\title{
1 Structure of human ferroportin bound to hepcidin reveals mechanisms of iron
}

\section{2 homeostasis}

4 Christian B. Billesbølle ${ }^{1 *}$, Caleigh M. Azumaya ${ }^{2 *}$, Rachael C. Kretsch ${ }^{3-7}$, Alexander S.

5 Powers $^{3-6,8}$, Shane Gonen ${ }^{2,9,10}$, Simon Schneider ${ }^{11}$, Tara Arvedson ${ }^{12}$, Ron O. Dror ${ }^{3-7}$, Yifan

6 Cheng $^{2,9}$, Aashish Manglik ${ }^{1,13}$

8 'Department of Pharmaceutical Chemistry, University of California, San Francisco, 1700 4th

9 Street, San Francisco, CA 94158, USA.

10 2Department of Biochemistry and Biophysics, University of California, San Francisco, 600 16th

11 Street, San Francisco, CA 94158, USA.

$12{ }^{3}$ Department of Computer Science, Stanford University, Stanford, CA 94305, USA

$13{ }^{4}$ Department of Molecular and Cellular Physiology, Stanford University School of Medicine,

14 Stanford, CA 94305, USA

$15{ }^{5}$ Department of Structural Biology, Stanford University School of Medicine, Stanford, CA 94305,

16 USA

$17{ }^{6}$ Institute for Computational and Mathematical Engineering, Stanford University, Stanford, CA

18 94305, USA

$19{ }^{7}$ Biophysics Program, Stanford University, Stanford, CA 94305, USA

$20{ }^{8}$ Department of Chemistry, Stanford University, Stanford, CA 94305, USA

$21{ }^{9}$ Howard Hughes Medical Institute, University of California San Francisco, San Francisco, CA,

22 USA

$23{ }^{10}$ Present address: Department of Molecular Biology and Biochemistry, University of California,

24 Irvine, 2224 Biological Sciences III, Irvine, CA 92697, USA

$25{ }^{11}$ Institute of Biochemistry, Goethe University Frankfurt, Max-von-Laue-Straße 9, 60438

26 Frankfurt am Main, Germany

$27{ }^{12}$ Department of Oncology Research, Amgen Inc., South San Francisco, CA, United States

$28{ }^{13}$ Department of Anesthesia and Perioperative Care, University of California, San Francisco,

291700 4th Street, San Francisco, CA 94158, USA.

31 * These authors contributed equally

32 Correspondence to Yifan Cheng (Yifan.Cheng@ucsf.edu) or Aashish Manglik

33 (Aashish.Manglik@ucsf.edu) 


\section{Abstract}

37 The serum iron level in humans is tightly controlled by the action of the hormone hepcidin on the

38 iron efflux transporter ferroportin. Hepcidin negatively regulates iron absorption and recycling by

39 inducing ferroportin internalization and degradation. Aberrant ferroportin activity can lead to

40 diseases of iron overload, like hemochromatosis, or iron limitation anemias. Here, we

41 determined cryogenic electron microscopy (cryo-EM) structures of ferroportin in lipid nanodiscs,

42 both in the apo state and in complex with cobalt, an iron mimetic, and hepcidin. These

43 structures and accompanying molecular dynamics simulations identify two divalent metal

44 binding sites within the $\mathrm{N}$ - and $\mathrm{C}$-domains of ferroportin. Hepcidin binds ferroportin in an

45 outward-open conformation and completely occludes the iron efflux pathway. The

46 carboxy-terminus of hepcidin directly contacts the divalent metal in the FPN C-domain. We

47 further show that hepcidin binding to ferroportin is coupled to iron binding, with an 80 -fold

48 increase in hepcidin affinity in the presence of iron. These results suggest a new model for

49 hepcidin regulation of ferroportin, where only iron loaded ferroportin molecules are targeted for

50 degradation. More broadly, our structural and functional insights are likely to enable more

51 targeted manipulation of the hepcidin-ferroportin axis in disorders of iron homeostasis. 
58 level. Iron disorders in humans can result from dysregulation of hepcidin or FPN, reflecting the central role of the hepcidin-FPN axis in iron homeostasis. Deficits in hepcidin-mediated regulation of FPN, often due to hereditary hemochromatoses, lead to iron overload and widespread tissue damage affecting the liver, pancreas, and joints ${ }^{11-13}$. By contrast, inappropriate elevation of hepcidin levels yields iron-restricted anemia ${ }^{14,15}$. Although several approaches to restore aberrant FPN function have been evaluated in clinical trials ${ }^{16-18}$, none have thus far succeeded.

9 The molecular mechanism of FPN regulation by hepcidin remains incompletely defined at the atomic level. A confluence of human genetics studies and structure-function evaluations have identified key regions of FPN important in hepcidin regulation ${ }^{6,19-22}$. A key recent advance was determination of the X-ray crystal structure of a divalent metal transporter from the bacterium Bdellovibrio bacteriovorus (bbFPN) with $40 \%$ similarity to human $\mathrm{FPN}^{23,24}$, which revealed a 84 unique architecture among the broader major facilitator superfamily (MFS) of membrane 
transporters. Although bbFPN is predicted to share structural features with human FPN, the precise mechanisms of iron coordination likely differ and bbFPN is not regulated by hepcidin.

To understand how FPN transports iron, and how this process is regulated by hepcidin, we used a combination of cryogenic electron-microscopy (cryo-EM), molecular dynamics simulations, and in vitro biochemical assays. These studies reveal the molecular recognition of iron and hepcidin by FPN and suggest a new regulatory mechanism enabling hepcidin to selectively target actively transporting ferroportin molecules for degradation.

\section{Structures of apo- and hepcidin-bound human FPN}

We screened the antigen-binding fragments (Fabs) of antibodies previously raised against $\mathrm{FPN}^{25}$ for use as a fiducial mark to guide image alignment of a small membrane protein embedded in a lipid nanodisc for structure determination by single particle cryo-EM, a strategy we proposed many years ago ${ }^{26}$. Among the many Fabs that bound purified FPN (Supplementary Fig. 1), a single clone, Fab45D8, yielded interpretable class averages in negative stain EM and was selected to facilitate cryo-EM structure determination. Unlike many antibodies and antibody fragments targeting FPN, Fab45D8 was previously determined to be non-competitive with hepcidin and, on its own, did not induce FPN internalization ${ }^{25}$. Indeed, in nanodisc-reconstituted preparations of FPN, Fab45D8 did not alter the binding properties of hepcidin (Supplementary Fig. 2).

We determined cryo-EM structures of nanodisc-reconstituted FPN bound to Fab45D8, both in the apo state (3.2 $\AA$, Supplementary Fig. 3) and bound to hepcidin and $\mathrm{Co}^{2+}(2.5 \AA$, Supplementary Fig. 4). We independently validated prior reports that FPN transports cobalt ${ }^{27}$ (Supplementary Fig. 1). Unlike $\mathrm{Fe}^{2+}, \mathrm{Co}^{2+}$ is not readily oxidized and therefore provides a tractable surrogate divalent metal for FPN biochemical and structural studies. The cryo-EM density map of FPN enabled building of an atomic model of FPN regions important for iron transport and hepcidin binding ${ }^{1,6}$, and a portion of the intracellular loop 3 (ICL3) important in hepcidin-induced FPN internalization ${ }^{8-10}$ (Supplementary Fig. 3 and 5a \& Supplementary Table 1). The entire FPN extracellular loop 5 (ECL5) remains unresolved, likely due to significant conformational flexibility. To enable modeling of Fab45D8, we separately obtained its X-ray crystal structure at $2.1 \AA$ (Supplementary Fig. 6 and Supplementary Table 2) 
Both cryo-EM structures reveal a monomeric FPN bound to a single Fab45D8 molecule, which recognizes a short alpha helical segment in FPN extracellular loop 2 (ECL2) (Fig. 1b and Supplementary Fig. 6). Similar to other MFS transporters, FPN contains twelve transmembrane (TM) helices arranged in two domains (Fig. 2a). Both the N-terminal and C-terminal domains are composed of six helices, with a large central cavity that, in both apo- and hepcidin-bound structures, is open to the extracellular side and closed intracellularly (Fig. 2b). Ferroportin shares significant structural similarity with the bacterial bbFPN transporter, with an overall root mean squared deviation (RMSD) of $2.0 \AA$ when compared to the outward-open conformation of bbFPN (Fig. 2c). The overall backbone conservation is even higher within the isolated C-terminal domain (RMSD 1.4 A) . Unlike most other MFS transporters, the alpha helix of FPN TM7 is interrupted by a short non-helical stretch of six residues. This unique feature, previously posited to be important in iron binding ${ }^{24}$, is shared between FPN and bbFPN.

Several interacting residues define an intracellular gate that keeps the $\mathrm{N}$ - and $\mathrm{C}$ - domains of FPN in an outward open conformation. Similar to a previously observed interaction network in bbFPN $^{23}, \mathrm{R} 489$ in TM11 of the C-domain forms an ionic interaction with D157 in TM4 of the $\mathrm{N}$-domain (Fig. 2d). This interaction is further supported by an extended ionic and hydrogen-bonding network including residues E486 (TM11) and R88 (TM3). In FPN, an additional cluster of ionic and hydrogen bonding interactions between TM5 in the N-domain and TM10 in the C-domain further stabilizes the outward open conformation (Fig. 2e). Mutation of several residues within the intracellular gate leads to FPN loss of function in ferroportin disease, highlighting the importance of the gate in coordinating the conformational steps necessary for iron efflux ${ }^{19,28}$

\section{Iron binds to the $\mathbf{N}$ and $\mathbf{C}$ domains of FPN}

Two distinct sites capable of binding divalent cations have previously been proposed for bbFPN. Although initial crystallographic studies suggested that iron primarily binds in a cavity within the $\mathrm{N}$ domain of the bbFPN transporter ${ }^{23}$, further mutagenesis studies found a critical divalent cation binding site within the $\mathrm{C}$ domain ${ }^{24}$. Structural elucidation of the FPN iron binding site, however, remains elusive. Previous studies on bbFPN either used supraphysiological concentrations of iron or found $\mathrm{Ni}^{2+}$ bound as an EDTA complex. 
150 We obtained cryo-EM data for a $\mathrm{Co}^{2+}$-hepcidin-FPN complex in the presence of a 8-fold molar excess of $\mathrm{CoCl}_{2}$ to $\mathrm{FPN}\left(100 \mu \mathrm{M} \mathrm{CoCl}_{2}: 12.5 \mu \mathrm{M} \mathrm{FPN}\right)$ to minimize artifacts arising from supraphysiological metal concentrations. Comparison of this map with the apo-FPN map revealed two new densities in the central cavity of FPN, corresponding to single metal binding sites in the $\mathrm{N}$ and in the $\mathrm{C}$ domain respectively (Fig. 3a-c). Within the $\mathrm{C}$ domain, $\mathrm{Co}^{2+}$ directly interacts with C326 in TM7b and H507 in TM11 while making a water-mediated contact with D325 and the backbone carbonyl of T320 (Fig. 3b). Intriguingly, the tetrahedral coordination geometry for $\mathrm{Co}^{2+}$ is fulfilled by the carboxy terminus of hepcidin. Within the $\mathrm{N}$ domain, we observe density for $\mathrm{Co}^{2+}$ coordinated directly by TM1 residues D39 and H43 (Fig. 3c).

We captured $\mathrm{Co}^{2+}$ bound to FPN in the presence of hepcidin. Hepcidin binding likely influences the structure and dynamics of FPN and it is therefore possible that the metal binding sites are different in FPN in the absence of hepcidin. To better understand divalent metal binding to FPN in the absence of hepcidin, we used all-atom molecular dynamics simulations. We first performed six simulations of apo-FPN in a hydrated lipid bilayer with $\mathrm{Fe}^{2+}$ ions initially positioned randomly in bulk solvent. In all six independent simulations, $\mathrm{Fe}^{2+}$ ions bound spontaneously to the $\mathrm{C}$ domain, localizing to the unwound region of TM7 near residues D325, D504, and H507 within hundreds of nanoseconds of simulation time (Fig. 3d and Supplementary Fig. 7). The Fe ${ }^{2+}$ ion also occasionally moves closer to TM1 to interact with D39, consistent with the additional $\mathrm{N}$-domain site observed in the hepcidin-bound structure. In parallel simulations of apo-FPN run without $\mathrm{Fe}^{2+}$ ions, we observed mobility of TM7b, with significant fluctuations of D325 (Fig. 3e). This observation is consistent with comparatively weaker cryo-EM density for TM7b as compared to other transmembrane helices in apo-FPN (Supplementary Fig. 7). By contrast, both D325 and TM7b are less mobile in simulations with iron bound at the $\mathrm{C}$ domain site (Fig. $3 e)$. Divalent metal binding to the $C$ domain may therefore stabilize an otherwise dynamic TM7b in a conformation that favors hepcidin-binding.

The identification of two metal binding sites within FPN raises the question of whether both sites are required for iron efflux. Several human FPN mutations that lead to hereditary hemochromatosis map to residues that directly coordinate $\mathrm{Co}^{2+}$ in the $\mathrm{C}$ domain, including C326S/F/Y $\mathrm{Y}^{13,29-31}$ and H507R ${ }^{32}$. Although these mutations likely disrupt the precise coordination geometry required for metal binding, they are fully competent to transport iron even in the presence of hepcidin; indeed, this lack of hepcidin-responsiveness leads to iron overload. 
183 Mutation of D325 leads to decreased iron efflux ${ }^{22}$, which initially suggested a key site for iron

184 efflux in the $\mathrm{C}$ domain of $\mathrm{FPN}^{24}$. However, recent modeling studies of an inward open

185 conformation of FPN based on bbFPN suggest that D325 may interact with the $\mathrm{N}$ domain; loss

186 of iron efflux in D325 mutants may therefore be caused by disruption of the extracellular gate ${ }^{33}$.

187 By contrast, mutation of D39 to alanine in the $\mathrm{N}$ domain metal binding site of FPN completely

188 abolishes iron efflux from HEK293 cells $^{22}$, suggesting that the $\mathrm{N}$ domain may be a primary site

189 for effluxed iron. Because the $\mathrm{C}$ domain metal-binding site is important for hepcidin binding, it

190 may primarily serve an important iron-dependent regulatory function in hepcidin control of FPN

191 activity.

193 Hepcidin occludes outward open FPN

194 Hepcidin binds FPN in a central cavity between the $\mathrm{N}$ and $\mathrm{C}$ domains, acting as a molecular

195 cork to completely occlude the iron efflux pathway (Fig. 1c and 4a). This structural observation

196 supports recent studies demonstrating acute inhibition of iron transport by hepcidin that is

197 independent of FPN ubiquitination and degradation ${ }^{6}$. Although the conformations of apo- and

$198 \mathrm{Co}^{2+}$-hepcidin-bound FPN are highly similar (RMSD $0.73 \AA$ ), the $\mathrm{Co}^{2+}$-hepcidin-bound structure

199 shows a $\sim 3 \AA$ rigid body separation of the $\mathrm{N}$ and $\mathrm{C}$ domains on the extracellular side of FPN

200 (Fig. 4b). Within the $\mathrm{N}$ domain, hepcidin binding leads to displacement of TM2 from the central

201 cavity, driven in part by a specific contact between I6 of hepcidin and T61 and Y64. Within the C

202 domain, the largest conformational changes occur around the $\mathrm{Co}^{2+}$ binding site, leading to

203 changes in the conformation of TM7b and the extracellular side of TM11 (Fig. 4b).

205 Hepcidin makes extensive polar and hydrophobic contacts with FPN with a total buried surface 206 area of $\sim 1300 \AA^{2}$. Our structure of hepcidin bound to FPN provides insight into disease-causing

207 mutations associated with FPN gain of function in hereditary hemochromatoses. Several FPN

208 mutations decrease hepcidin binding to FPN, including N144H/D/T ${ }^{34-36}, C 326 S / F / Y^{13,29-31}$,

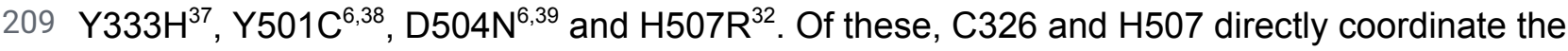

210 cobalt ion and mutations therefore indirectly affect hepcidin affinity or alter the atomic basis of

211 binding specificity. Other interactions between FPN and hepcidin are either hydrophobic or

212 depend on hepcidin amide backbone atoms, which is consistent with the relatively high

213 tolerance of amino acid substitutions within hepcidin ${ }^{40}$. For example, Y333 fits into a

214 hydrophobic cavity in hepcidin and hydrogen bonds with the backbone carbonyl of hepcidin

215 residue M21 (Fig. 4c). D504 in FPN coordinates the backbone amide of hepcidin H3 while Y501 
$216 \pi$-stacks with the imidazole side chain of H3 (Fig. 4c). A further hydrogen bond between N144

217 and $\mathrm{Y} 501$ further stabilizes this interaction network. In contrast to these mutations, the $\mathrm{Y} 64 \mathrm{~N} / \mathrm{H}$

218 mutants retain hepcidin binding $^{6}$, but are completely resistant to hepcidin-induced FPN

219 ubiquitination $^{41,42}$. The outward displacement of TM2 near Y64 induced by hepcidin may

220 therefore be important for FPN ubiquitination (Fig. 4c).

222 The structure of hepcidin bound to FPN provides insight into prior efforts to engineer hepcidin

223 mimetics as potential therapeutics for diseases of iron overload $20,40,43,44$. Although the first two

224 residues of hepcidin (DT) are dispensable for activity, residues 3-8 (HFPICl) are absolutely

225 required for function ${ }^{20}$. Indeed, the first two residues of hepcidin make minimal interactions with

226 FPN (Fig. 4c). By contrast, alanine scanning mutagenesis suggested an important role for

227 hydrophobic hepcidin residues including F4 and F9, and to a lesser extent $\mathrm{H} 3$ and $16^{40,43,44}$.

228 Residues F4 and F9 insert between the $\mathrm{N}$ and C domains of FPN at opposite ends of the central

229 cavity (Fig. 4c); hydrophobic residues at these positions likely stabilize the FPN outward open

230 state. More unexpected is the extensive set of contacts between hepcidin residues 10-25 and

231 TM7b of FPN. Prior studies have recapitulated hepcidin activity with a linear peptide composed

232 of hepcidin residues 1-9, though with a $\sim 8-20$ fold reduction in potency ${ }^{40,45}$. Whether these

233 minihepcidins fully plug the iron efflux pathway remains unclear. Furthermore, in the absence of

234 structural data, it remains unclear whether minihepcidin variants coordinate divalent metals in

235 the same manner as observed for hepcidin in our structure.

237 In addition to blocking the iron transport pathway, hepcidin regulates FPN by causing

238 ubiquitination of lysine residues in intracellular loop 3 (ICL3) ${ }^{9,10}$. Among these, K240 is critical for

239 hepcidin-induced FPN internalization and degradation. Neither the apo- or $\mathrm{Co}^{2+}$-hepcidin-bound

240 structures resolve residues 239-288 of ICL3, precluding a structural understanding of how

241 hepcidin regulates the conformation of K240. In the resolved regions of ICL3, we observe no

242 significant conformational changes between apo- and $\mathrm{Co}^{2+}$-hepcidin-bound FPN (Fig. 4d,e).

243 Several caveats may limit our structural analysis of hepcidin-induced conformational changes in

244 FPN, including the lack of a membrane voltage or proton gradient across the lipid nanodisc, the

245 requirement for specific lipids for FPN function, or other cofactors important in hepcidin-induced

246 FPN ubiquitination. However, the structures provide clues into the role of ICL3 in FPN function.

247 The $\mathrm{N}$ terminal portion of ICL3 (residues 230-238) forms interactions with the $\mathrm{N}$ domain (Fig.

248 4d). Notably, K236 makes an ionic interaction with the intracellular gate residue D157. The C 
249 terminal portion of ICL3 (residues 291-304) forms an amphipathic helix that makes a number of

250 contacts with both TM2 in the $\mathrm{N}$ domain and TM11 in the $\mathrm{C}$ domain, which both undergo

251 conformational changes upon binding hepcidin (Fig. 4e). In both cases, the resolved regions of

252 ICL3 are primed to sense the conformation of the transporter as it shuttles iron and binds

253 hepcidin. These regions may therefore serve as important conduits linking the conformation of

254 hepcidin binding on the extracellular side to K240 conformation on the intracellular side.

\section{Hepcidin is coupled to iron binding}

257 The direct interaction between hepcidin and $\mathrm{Co}^{2+}$ in our structure is unexpected (Fig. 5a), and

258 suggests that divalent metals may be important for hepcidin binding to FPN. We therefore

259 directly tested the effect of $\mathrm{Fe}^{2+}$ and $\mathrm{Co}^{2+}$ on hepcidin affinity at FPN. A fluorescently tagged

260 version of hepcidin (Rhodamine green-hepcidin ${ }^{25}$, RhoG-Hep) bound to nanodisc-reconstituted

261 ferroportin with an apparent $\mathrm{K}_{\mathrm{D}}$ of $210 \mathrm{nM}\left(\mathrm{pK}_{\mathrm{D}}=-6.67 \pm 0.02\right)$ (Fig. 5b). In the presence of 10

$262 \mu \mathrm{M} \mathrm{FeCl}_{2}$, we observed a significantly increased affinity of $2.5 \mathrm{nM}\left(\mathrm{pK}_{\mathrm{D}}=-8.61 \pm 0.21\right)$, an

263 almost 80-fold change in the potency of hepcidin at FPN. Addition of $\mathrm{CoCl}_{2}$ also increased

264 hepcidin affinity. Consistent with a cooperative effect, the effect of $\mathrm{CoCl}_{2}$ on hepcidin binding

265 was saturable (Fig. 5c).

267 The reference range for hepcidin concentration in healthy adults is $\sim 1-30 \mathrm{nM}^{46}$. Our in vitro

268 binding experiment with purified FPN indicates minimal hepcidin binding to FPN in the absence

269 of a divalent metal, suggesting that metal binding to FPN may regulate hepcidin activity in vivo.

270 Our structure of FPN bound to $\mathrm{Co}^{2+}$ and hepcidin revealed conformational changes in TM7b

271 associated with hepcidin binding (Fig. 3e) and a direct contact between hepcidin and $\mathrm{Co}^{2+}$ (Fig.

$2723 b)$; both could be important for the observed effect of $\mathrm{Fe}^{2+}$ and $\mathrm{Co}^{2+}$ on hepcidin binding. We

273 therefore tested whether disruption of the $C$ domain iron binding site influences hepcidin

274 binding. Even in the presence of $50 \mu \mathrm{M} \mathrm{CoCl}_{2}$, the D325N, C326S, and H507R mutants bound

275 hepcidin weakly, titrating in a micromolar range similar to wild-type FPN in the absence of

276 divalent metals (Fig. 5c). These results highlight the critical role of the $\mathrm{C}$ domain metal site in

277 potent hepcidin binding to FPN, which is likely important in homeostatic control of iron levels in a

278 physiological setting. 
281 Ferroportin is a central regulator of iron homeostasis in humans. Both human FPN and the

282 bacterial homologue bbFPN show remarkable similarities in their overall architecture, with a

283 unique conformation of TM7 responsible for molecular recognition of iron within the C domain.

284 This similarity suggests a deep evolutionary history for FPN-like transporters within the broader

285 major facilitator superfamily. Hepcidin, by contrast, is specific to vertebrates and likely evolved

286 as a new strategy to regulate a critical point in iron absorption. The structures presented here

287 map, at high resolution, metal and hepcidin binding to FPN.

289 We determined that hepcidin binding to FPN is greatly potentiated by iron itself, potentially due

290 to the stabilizing effect iron has on the hepcidin-binding site of FPN (Fig. 5d). With iron, the

291 binding affinity of hepcidin falls in a range concordant with the concentration of hepcidin

292 observed in healthy human adults. In normal iron homeostasis, this may enable hepcidin to

293 selectively bind and regulate FPN molecules actively transporting iron and loaded with $\mathrm{Fe}^{2+}$,

294 while sparing FPN molecules located on cells with low transport activity. Hepcidin binding to

295 FPN would both trap the transporter in an outward open state and limit iron egress; both actions

296 acutely decrease iron efflux, as has been recently reported ${ }^{6}$. Elevated hepcidin levels likely

297 inappropriately overcome this regulatory strategy and degrade FPN even in the absence of

298 active iron efflux. The potentiation of hepcidin activity by iron may therefore have immediate

299 consequences for the development of hepcidin mimetics currently in clinical trials ${ }^{16}$.

300 Furthermore, hepcidin antagonism by direct targeting of FPN may require molecules with high

301 potency to overcome the nanomolar effect of the hormone in the presence of iron. The structural

302 and functional insights into FPN function presented here therefore provide critical foundations

303 for the discovery of therapeutics for human disorders of iron homeostasis. 


\section{MAIN TEXT FIGURES}

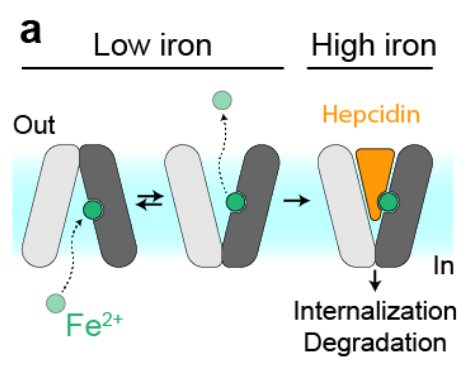

C apo-FPN

$\mathbb{N}$ domañ C domain

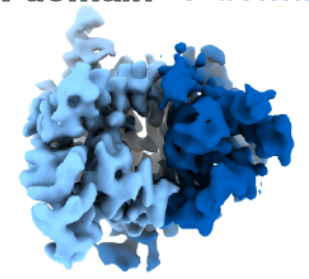

FPN:Co ${ }^{2+}:$ hepcidin
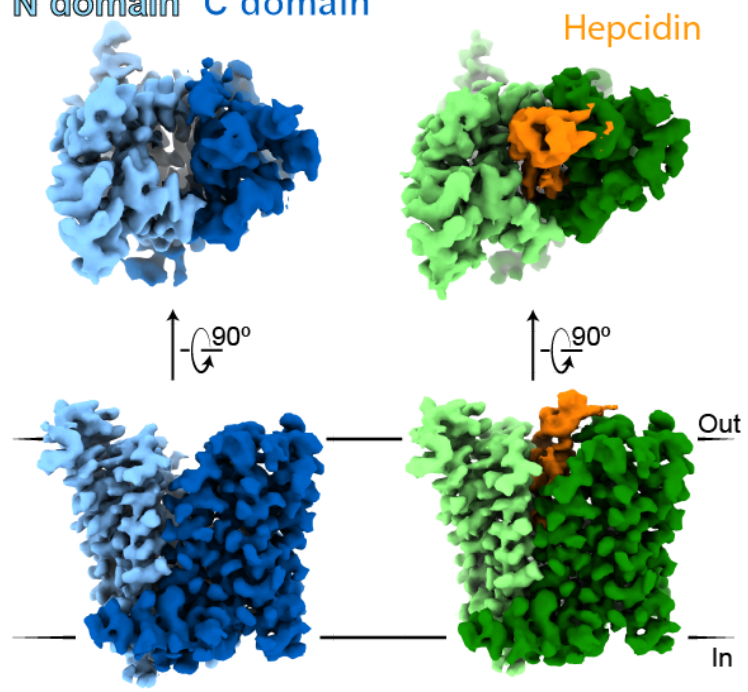

309 Figure 1. Structures of human ferroportin. a, Ferroportin effluxes cellular iron $\left(\mathrm{Fe}^{2+}\right)$ by an alternating access mechanism. Hepcidin binds to outward open ferroportin and induces ubiquitination and degradation. b, Cryo-EM map of apo-FPN-Fab45D8 complex in lipid nanodisc. c, Cryo-EM density of apo and $\mathrm{Co}^{2+} /$ hepcidin bound FPN. The $\mathrm{N}$ and $\mathrm{C}$ domains are colored in different shades of blue for apo-FPN and green for $\mathrm{Co}^{2+} /$ hepcidin bound FPN. Hepcidin (orange) binds to an extracellular facing cavity in FPN. 
a
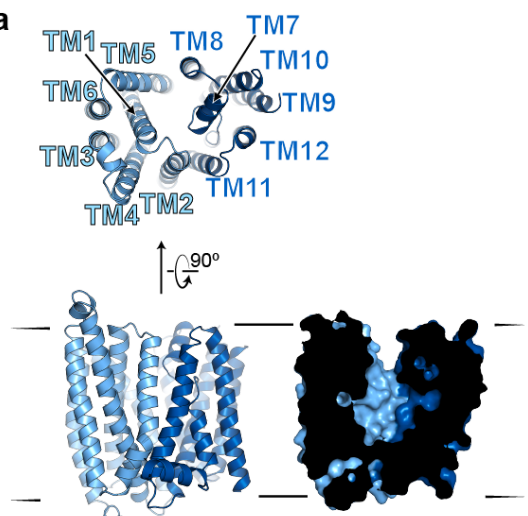

$\mathbb{N}$ domân $\mathrm{C}$ domain

b

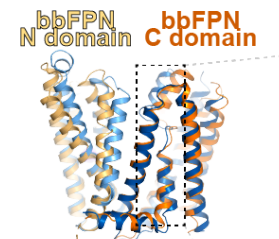

C

bbFPN outward open

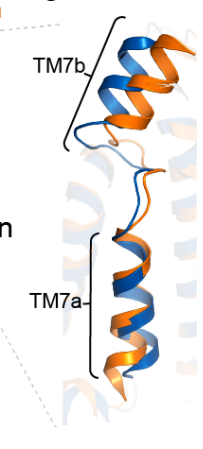

bbFPN inward open

d

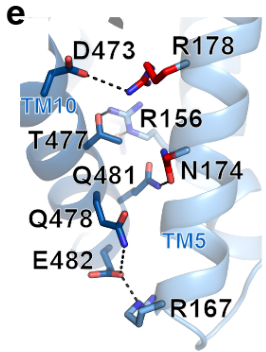

Figure 2. Structure of apo-FPN and similarity to bbFPN. a, Ribbon diagram of FPN reveals 12 transmembrane helices. The $\mathrm{N}$ - and $\mathrm{C}$-domains are colored in different shades of blue. Cutaway surface view (right) shows outward open conformation. b, Human FPN aligned to the outward-open (PDB: 5AYN) and inward-open (PDB: 5AYO) conformations of bbFPN. c, Unique architecture of TM7 shared between human FPN and bbFPN. d, Intracellular gating residues are shown as sticks. Residues in red are known FPN loss-of-function mutations e, TM10 and TM5 form an extensive network of interactions, further stabilizing the outward open conformation. Residues highlighted in red are known loss-of-function mutations that lead to ferroportin disease in humans. 


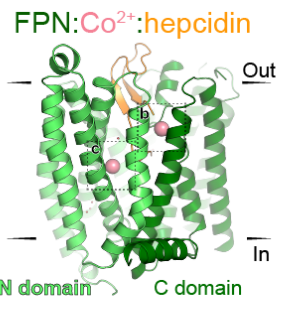

d

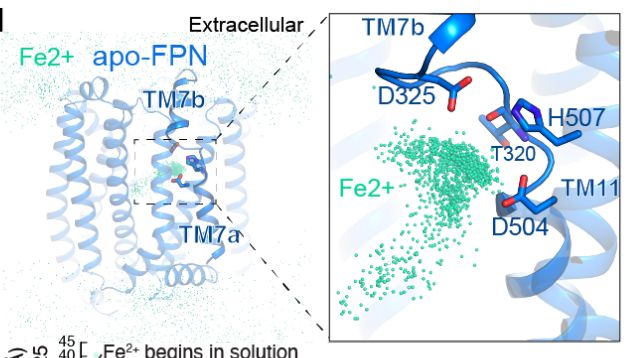

b
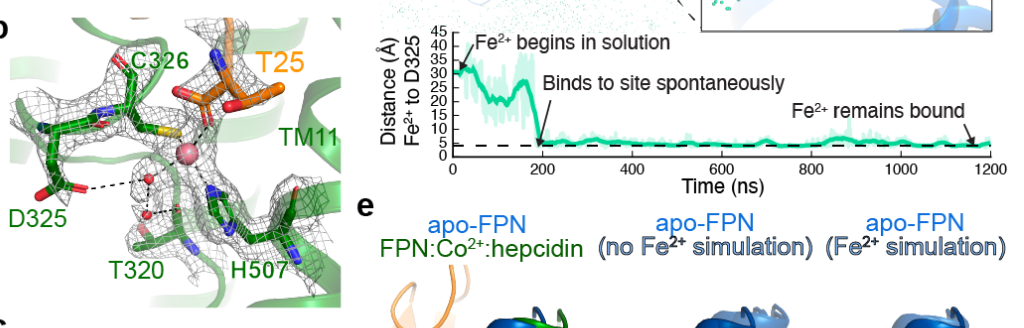

C
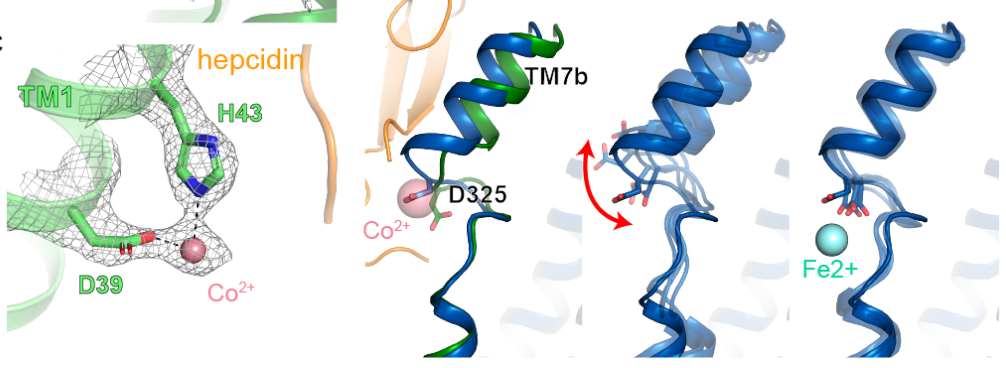

Figure 3. Iron binds to the $\mathbf{N}$ and $\mathbf{C}$ domains of FPN. a, Ribbon diagram of view of cryo-EM density for $\mathrm{Co}^{2+}$ ion in the FPN C domain. $\mathrm{Co}^{2+}$ binds with tetrahedral coordination to $\mathrm{C} 326, \mathrm{H} 507$, a water molecule, and the hepcidin C-terminus. c, Closeup view of cryo-EM density for $\mathrm{Co}^{2+}$ ion in the FPN N domain, coordinated by $\mathrm{H} 43$ and D39. d, In molecular dynamics simulations with $\mathrm{Fe}^{2+}$ initially positioned randomly in bulk water surrounding $\mathrm{FPN}$, the $\mathrm{Fe}^{2+}$ ions spontaneously bind to a region near $\mathrm{H} 507, \mathrm{D} 325$, and D504. The aggregated position of $\mathrm{Fe}^{2+}$ ions from six simulations, each $2 \mu \mathrm{s}$ in length, is shown superimposed with apo FPN. c, In one representative simulation, an $\mathrm{Fe}^{2+}$ ion spontaneously binds within $200 \mathrm{~ns}$ and remains localized at this site for more than $1000 \mathrm{~ns}$. Distance shown is from the ion to the nearest oxygen atom of the D325 side chain. Thick trace represents a 15-ns sliding mean and thin traces represent unsmoothed values. e, Comparison of TM7b conformation in apo-FPN and FPN bound to $\mathrm{Co}^{2+}$ and hepcidin. In simulations without $\mathrm{Fe}^{2+}, \mathrm{TM} 7 \mathrm{~b}$ is dynamic, with significant fluctuation of D325. D325 coordinates $\mathrm{Fe}^{2+}$ in simulations, and is associated with decreased 


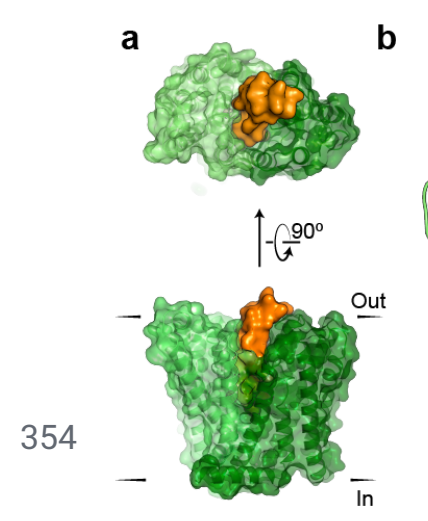

d

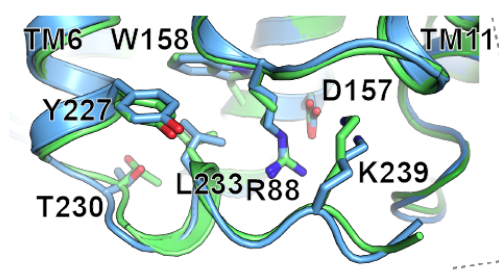

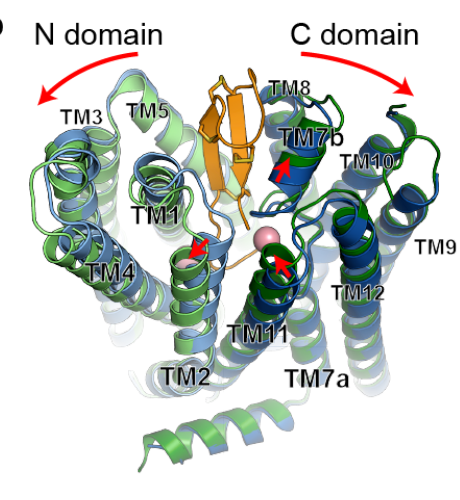

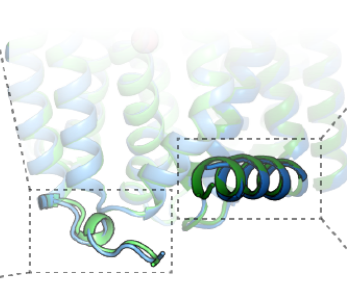

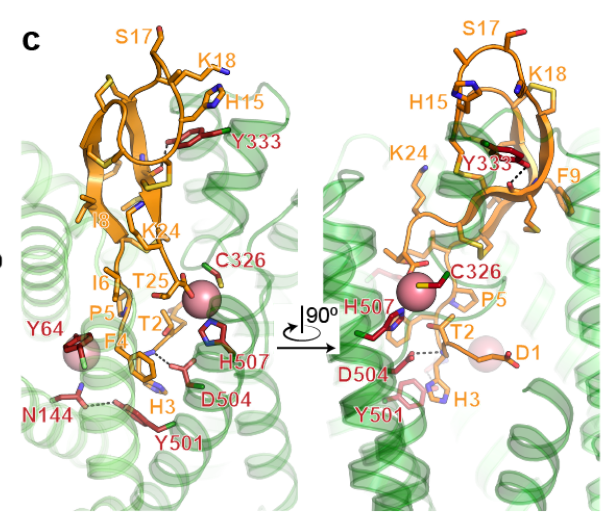

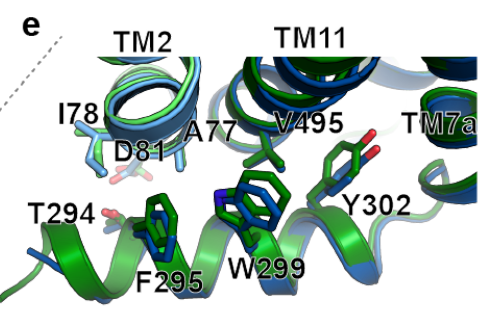

355 Figure 4. Hepcidin binding to FPN. a, Surface representation of the FPN-Co ${ }^{2+}$-hepcidin complex viewed from the extracellular side and perpendicular to the membrane plane. $\mathbf{b}$, Ribbon diagrams of apo-FPN (blue) aligned to FPN-Co ${ }^{2+}$-hepcidin (green, orange, and pink spheres) showing the overall separation of the $\mathrm{N}$ and $\mathrm{C}$ domains, and the displacement of TMs containing key residues for hepcidin binding. c, Close-up views of the hepcidin binding site. Residues in red are known hepcidin resistance mutations involved in ferroportin disease. d,e Close-up of interactions between the transmembrane regions and intracellular loop 3. 
a

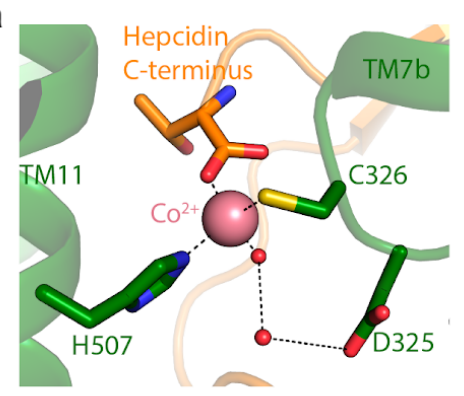

b

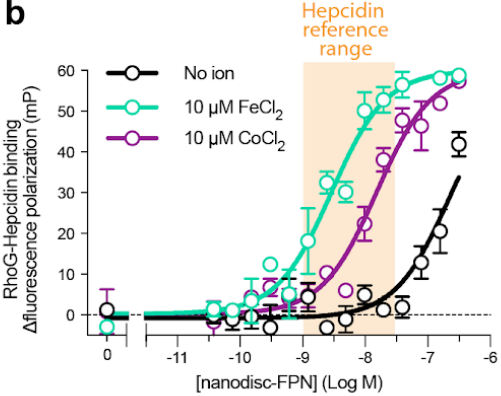

C

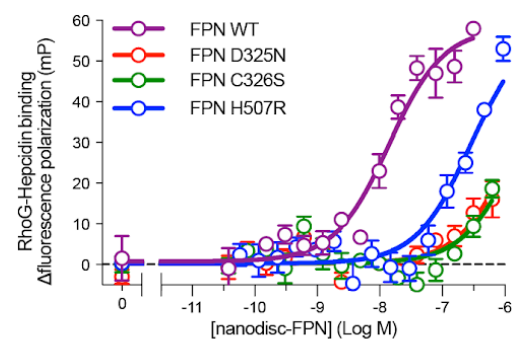

362
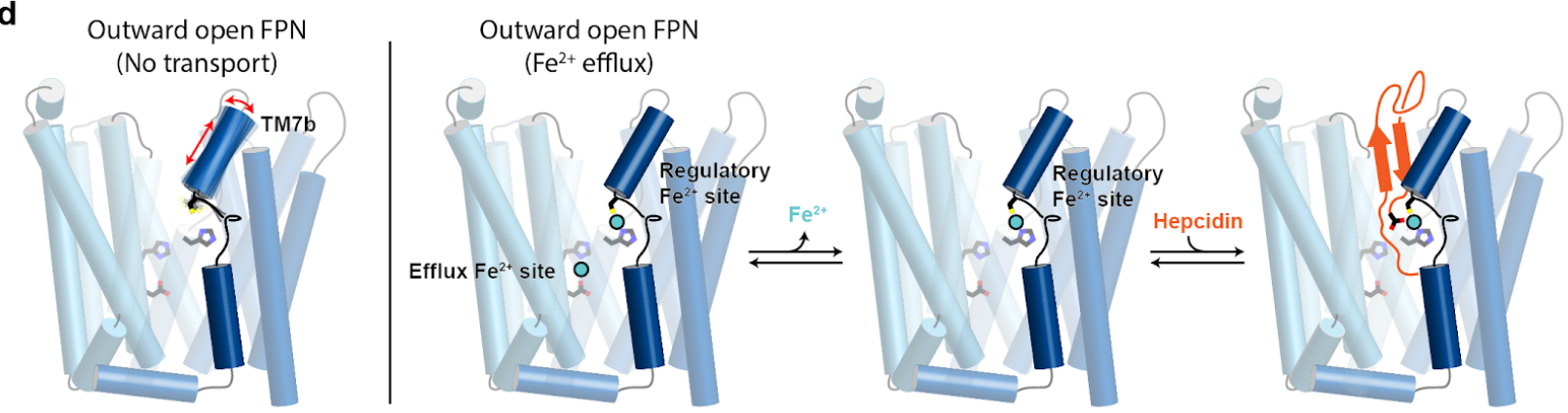

363 Figure 5. Hepcidin binding is potentiated by iron. a, FPN C domain metal binding site.

364 Shown are residues that interact with $\mathrm{Co}^{2+}$, including D325, C326, and $\mathrm{H} 507$ in FPN as well as

365 the hepcidin C-terminus. b, Fluorescence polarization increase in rhodamine green-labeled hepcidin (RhoG-hepcidin) as nanodisc-reconstituted FPN is titrated with a $\mathrm{K}_{\mathrm{D}}$ of $210 \mathrm{nM}$. Addition of $10 \mu \mathrm{M} \mathrm{FeCl}_{2}$ or $\mathrm{CoCl}_{2}$ increases the affinity of hepcidin to $2.5 \mathrm{nM}$ and $7.7 \mathrm{nM}\left(\mathrm{pK}_{\mathrm{D}}=\right.$ $-8.11 \pm 0.16)$, respectively. Hepcidin concentration range in healthy human adults is shown in orange. c, Mutation of the $\mathrm{C}$ domain metal binding sites decreases RhoG-hepcidin binding affinity at $\mathrm{FPN}$, even in the presence of $50 \mu \mathrm{M} \mathrm{CoCl}_{2}$. All values are reported as mean \pm s.e.m. Error bars represent s.e.m. d, Model for iron-coupled hepcidin regulation of FPN function. In settings of iron efflux, TM7b is conformationally stabilized by iron coordination in the $\mathrm{C}$ domain regulatory site. High affinity hepcidin binding to outward open FPN depends on the direct coordination of iron in the $\mathrm{C}$ domain. 


\section{METHODS}

\section{Expression and purification of human ferroportin}

384 The wild-type human FPN gene was cloned into a pVL1392 vector containing an expression

385 cassette comprised of a $\mathrm{C}$ terminal human rhinovirus 3C (HRV-3C) protease recognition

386 sequence followed by a human protein $\mathrm{C}$ epitope tag (EDQVDPRLIDGK) and an 8x polyhistidine tag. Baculovirus was generated using Spodoptera frugiperda Sf9 insect cells (unauthenticated and untested for mycoplasma contamination, Expression Systems 94-001F) and the construct was expressed in Spodoptera frugiperda Sf9 insect cells. Cells were collected $48 \mathrm{~h}$ after transduction and stored at $-80^{\circ} \mathrm{C}$ until further use. Frozen cell pellets were thawed and washed with a hypotonic buffer (20 mM HEPES pH 7.50, 1 mM EDTA, supplemented with $20 \mu \mathrm{g} / \mathrm{mL}$ leupeptin, and $160 \mu \mathrm{g} / \mathrm{mL}$ benzamidine) before solubilizing with $50 \mathrm{mM}$ HEPES $\mathrm{pH}$ 7.5, $300 \mathrm{mM} \mathrm{NaCl}, 1 \%$ (w/v) n-dodecyl- $\beta$-D-maltopyranoside (DDM, Anatrace), $0.1 \%$ (w/v) cholesteryl hemisuccinate (CHS, Steraloids), $1 \mathrm{mM}$ EDTA supplemented with $20 \mu \mathrm{g} / \mathrm{mL}$ leupeptin, and $160 \mu \mathrm{g} / \mathrm{mL}$ benzamidine for $1 \mathrm{~h}$ at $4^{\circ} \mathrm{C}$. Following centrifugation, the resulting supernatant was loaded on homemade anti-protein C antibody Sepharose beads and washed extensively in $50 \mathrm{mM}$ HEPES pH 7.50, $300 \mathrm{mM} \mathrm{NaCl}, 2 \mathrm{mM} \mathrm{CaCl}, 0.1 \%$ (w/v) DDM, 0.01\% (w/v) CHS. FPN was eluted with $50 \mathrm{mM}$ HEPES pH 7.50, $300 \mathrm{mM} \mathrm{NaCl}, 0.1 \%$ (w/v) DDM, $0.01 \%(w / v) ~ C H S, 0.2 \mathrm{mg} / \mathrm{mL}$ Protein C peptide (Genscript) and $5 \mathrm{mM}$ EDTA. The protein was concentrated with a Vivaspin 100-kDa MWCO concentrator and the monomeric FPN fraction was collected after size-exclusion chromatography (SEC) over a Superdex S200 Increase 10/300 GL column (GE Healthcare) equilibrated with $20 \mathrm{mM} \mathrm{HEPES} \mathrm{pH} \mathrm{7.50,100} \mathrm{mM} \mathrm{NaCl} \mathrm{and}$ $0.1 \%(w / v)$ DDM, and $0.01 \%(w / v)$ CHS.

\section{Expression and purification of MSP}

Constructs encoding MSP-NW9 or MSP-NW11 ${ }^{47}$ in the pET28b vector (Addgene \#133442) were transformed into BL21(DE3) Rosetta Escherichia coli, and grown in terrific broth medium supplemented with $2 \mathrm{mM} \mathrm{MgCl}_{2}$ and $0.1 \%(\mathrm{w} / \mathrm{v})$ glucose at $37^{\circ} \mathrm{C}$. At $\mathrm{OD}_{600}$ of $\sim 0.6$, expression was induced by addition of $400 \mu \mathrm{M}$ isopropyl $\beta$-d-1-thiogalactopyranoside (IPTG) and lowering the temperature to $20^{\circ} \mathrm{C}$. Cells were harvested after 16 hours and resuspended into $5 \mathrm{~mL}$ lysis 
buffer (200 mM Tris pH 8.0, 500 mM NaCl, 1\% (v/v) Triton X-100 (Sigma), 0.02 mg/mL leupeptin, $0.16 \mathrm{mg} / \mathrm{mL}$ benzamidine, and benzonase) per gram pellet. After stirring for $30 \mathrm{~min}$ at $4^{\circ} \mathrm{C}$, cells were lysed by pulsed sonication on ice. The lysate was cleared by centrifugation at $15,000 \times \mathrm{g}$ for $25 \mathrm{~min}$ at $4^{\circ} \mathrm{C}$ and loaded on Ni-NTA Sepharose. Ni-NTA beads were washed with $50 \mathrm{mM}$ Tris $\mathrm{pH} 8.0,500 \mathrm{mM} \mathrm{NaCl}, 1 \%(\mathrm{v} / \mathrm{v})$ Triton, then $50 \mathrm{mM}$ Tris pH 8.0, $500 \mathrm{mM} \mathrm{NaCl}$, $50 \mathrm{mM}$ sodium cholate, then $50 \mathrm{mM}$ Tris $\mathrm{pH} 8.0,500 \mathrm{mM} \mathrm{NaCl}$, and finally with $50 \mathrm{mM}$ Tris $\mathrm{pH}$ 8.0, $500 \mathrm{mM} \mathrm{NaCl}, 30 \mathrm{mM}$ Imidazole. MSP was eluted with $50 \mathrm{mM}$ Tris- $\mathrm{HCl} \mathrm{pH}$ 8.0, $500 \mathrm{mM}$ $\mathrm{NaCl}, 400 \mathrm{mM}$ Imidazole and dialyzed into $50 \mathrm{mM}$ Tris- $\mathrm{HCl}, \mathrm{pH}$ 8.0, $100 \mathrm{mM} \mathrm{NaCl}, 1 \mathrm{mM}$ EDTA, $0.1 \mathrm{mM}$ TCEP at $4^{\circ} \mathrm{C}$. The following day, MSP was concentrated on a Vivaspin 10-kDa MWCO concentrator, aliquots were flash frozen in liquid nitrogen and stored at $-80^{\circ} \mathrm{C}$ for reconstitution.

\section{Isolation, expression and purification of Fab45D8}

The heavy and light chain sequences of $\mathrm{mAb} 45 \mathrm{D} 8^{25}$ were separately cloned into pcDNA3.4 and the resulting vectors were transfected into Expi293F Human Embryonic Kidney cells (Life Technologies) using a 1:2 mass ratio of light and heavy chain DNA with the Expifectamine transfection kit (Life Technologies) as per the manufacturer's instructions. Supernatant containing mAb45 was harvested $136 \mathrm{~h}$ after transfection and loaded on homemade Protein G Sepharose beads and extensively washed with a buffer comprising $20 \mathrm{mM} \mathrm{HEPES} \mathrm{pH} \mathrm{7.50,}$ and $100 \mathrm{mM} \mathrm{NaCl}$. mAb45D8 was eluted with $100 \mathrm{mM}$ glycine ( $\mathrm{pH}$ 3.0) and fractions were immediately neutralized with $200 \mathrm{mM}$ HEPES pH 7.50. To generate the Fab fragment, $10 \mathrm{mg}$ of purified mAb45D8.1 was diluted into $9.5 \mathrm{ml}$ freshly prepared cleavage buffer ( $20 \mathrm{mM}$ sodium phosphate $\mathrm{pH} 7.00,10 \mathrm{mM}$ EDTA, and $10 \mathrm{mM}$ cysteine) and treated with $0.5 \mathrm{ml}$ agarose immobilized papain (Thermo Scientific) at $37^{\circ} \mathrm{C}$. After $16 \mathrm{~h}$ the cleaved Fab45D8 fragment was purified by reverse Protein A affinity chromatography, followed by SEC into buffer comprising 20 mM HEPES pH 7.50 and 100 mM NaCl. Fab45D8 was concentrated on a Vivaspin 10-kDa MWCO concentrator, and aliquots were flash frozen in liquid nitrogen and stored at $-80^{\circ} \mathrm{C}$ for later use.

\section{Reconstitution of FPN into lipidic nanodisc}

Purified FPN (0.2-0.5 mg) was mixed with purified MSP and a lipid mixture containing a 2:3 weight ratio of 1-palmitoyl-2-oleoylphosphatidylcholine (POPC, Avanti) and 1-palmitoyl-2-oleoyl-sn-glycero-3-phospho-(1'-rac-glycerol) (POPG, Avanti). For reconstitution 
444 into NW9 nanodiscs, an FPN:MSP:Lipid molar ratio of 1:20:1100 was used. For reconstitution

445 into NW11 nanodiscs, an FPN:MSP:Lipid molar ratio of 1:20:800 was used. The reconstitution

446 sample was nutated for $1 \mathrm{~h}$ at $4^{\circ} \mathrm{C}$ before addition of $0.2 \mathrm{~g} / \mathrm{mL} \mathrm{SM2-BioBeads} \mathrm{(BioRad),} \mathrm{and} \mathrm{the}$

447 reconstitution sample was further nutated overnight at $4^{\circ} \mathrm{C}$ before removal of the biobeads. FPN

448 containing nanodiscs were purified by loading the reconstitution sample on anti-protein $\mathrm{C}$

449 antibody Sepharose beads and washing extensively with $20 \mathrm{mM} \mathrm{HEPES} \mathrm{pH} \mathrm{7.50,100} \mathrm{mM}$

$450 \mathrm{NaCl}$, and $1 \mathrm{mM} \mathrm{CaCl}_{2}$ to remove empty nanodiscs. FPN containing nanodiscs were eluted with

$45120 \mathrm{mM}$ HEPES pH 7.50, $100 \mathrm{mM} \mathrm{NaCl}, 0.25 \mathrm{mM}$ EDTA, and $0.2 \mathrm{mg} / \mathrm{mL}$ Protein C peptide

452 (Genscript), and concentrated on a Vivaspin 50-kDa concentrator.

454 Crystallization and structure determination of Fab45D8

455 Purified Fab45D8 was diluted to $13.0 \mathrm{mg} / \mathrm{mL}$ in $20 \mathrm{mM} \mathrm{HEPES} \mathrm{pH} \mathrm{7.5,} 100 \mathrm{mM} \mathrm{NaCl}$. Fab45D8

456 crystals were obtained in $0.3 \mathrm{M}$ trimethylamine-N-oxide (TMAO), $0.1 \mathrm{M}$ Tris pH 8.5, and 30\%

457 (w/v) PEG $2000 \mathrm{MME}$ at $20^{\circ} \mathrm{C}$. Individual crystals were flash frozen in liquid nitrogen after a 30

458 s soak in $0.3 \mathrm{M}$ trimethylamine-N-oxide (TMAO), $0.1 \mathrm{M}$ Tris $\mathrm{pH} 8.5$, and $30 \%$ (w/v) PEG 2000,

459 and $20 \% \mathrm{v} / \mathrm{v}$ ethylene glycol. A full diffraction dataset was collected at the Advanced Photon

460 Source GM/CA-CAT beamline 23ID-B, and processed using xia2dials ${ }^{48}$ implementation of

$461 \mathrm{XDS}^{49}$. The structure of the Fab was solved by molecular replacement using Phaser ${ }^{50}$, with a

462 search model of a closely related germline mouse monoclonal antibody (PDB ID: 6BZV ${ }^{51}$ )

463 lacking complementarity determining regions (CDRs). The model was iteratively improved by

464 refinement in $\operatorname{Coot}^{52}$ and Phenix ${ }^{53}$. Data collection and refinement statistics are summarized in

465 Supplementary Table 1. The final model contained $96.77 \%, 2.23 \%$ and $0 \%$ in the favored,

466 allowed and outlier regions of the Ramachandran plot, respectively as assessed by

467 MolProbity ${ }^{54}$.

468

469 Calcein transport assay for divalent cations

470 FPN was reconstituted into liposomes for divalent cation transport assays. Empty liposomes

471 were prepared as a 3:1 mass ratio of 1-palmitoyl-2-oleoyl-sn-glycero-3-phosphoethanolamine

472 (POPE, Avanti) to POPG dissolved in chloroform, followed by gentle evaporation of the

473 chloroform under a stream of nitrogen gas, and overnight desiccation. The lipids were dissolved

474 in $20 \mathrm{mM}$ HEPES pH 7.40, $100 \mathrm{mM} \mathrm{KCl}$ to a final concentration of $12.5 \mathrm{mg} / \mathrm{mL}$, sonicated until

475 optically clear, subjected to multiple freeze-thaw cycles, and extruded through a $400 \mathrm{~nm}$

476 polycarbonate filter (Avestin) to generate unilamellar vesicles. Subsequently $0.13 \%(\mathrm{w} / \mathrm{v})$ 
477 Triton-X100 (Sigma) was added to destabilize liposomes, corresponding to the concentration

478 yielding $80 \%$ of the maximum $\mathrm{OD}_{540}$ obtained in a liposome destabilization curve. Purified FPN

479 was added at a 1:50 protein to lipid mass ratio and incubated for $15 \mathrm{~min}$ at $4^{\circ} \mathrm{C}$. Control

480 liposomes devoid of FPN were prepared in parallel using the same concentration of DDM. To

481 remove excess detergent, $0.05 \mathrm{~g} / \mathrm{mL}$ of SM2-BioBeads were added to the sample and nutated

482 for $1 \mathrm{hr}$ at $4^{\circ} \mathrm{C}$, then $0.05 \mathrm{~g} / \mathrm{mL}$ SM2-BioBeads were added followed by incubation overnight at

$4834^{\circ} \mathrm{C}$, and finally addition of $0.08 \mathrm{~g} / \mathrm{mL}$ SM2-BioBeads followed by incubation for $2 \mathrm{hr}$ at $4^{\circ} \mathrm{C}$.

484 Proteoliposomes were harvested by ultracentrifugation at $300,000 \times \mathrm{g}$ for $30 \mathrm{~min}$ and

485 resuspended at a concentration of $2.0 \mathrm{mg} / \mathrm{mL}$ lipids in internal buffer comprised of $20 \mathrm{mM}$

486 HEPES pH 7.40 and $100 \mathrm{mM} \mathrm{KCl}$, before flash freezing in liquid nitrogen and storage at $-80^{\circ} \mathrm{C}$.

487 On the day of the transport assay, proteoliposomes were thawed and incubated with $500 \mathrm{mM}$

488 calcein (Sigma), then subjected to three freeze-thaw cycles, and extruded through a $400 \mathrm{~nm}$

489 polycarbonate filter. The liposomes were washed four times with external buffer comprising 20

$490 \mathrm{mM}$ HEPES pH 7.40 and $100 \mathrm{mM} \mathrm{NaCl}$, by repeated ultracentrifugation and resuspension.

491 Immediately prior to the assay, the calcein containing proteoliposomes were diluted to 0.25

$492 \mathrm{mg} / \mathrm{mL}$ lipid in external buffer. Time-course fluorescence traces were recorded as $1 \mathrm{~s}$

493 integrations using a FluoroMax-4 (Horiba) with $\lambda_{\mathrm{ex}}$ of $490 \mathrm{~nm}$ and $\lambda_{\mathrm{em}}$ of $520 \mathrm{~nm}$. Steady state

494 fluorescence was recorded for at least $5 \mathrm{~min}$, before addition of small aliquots of freshly

495 prepared stocks of either $\mathrm{FeCl}_{2}$ or $\mathrm{CoCl}_{2}$. To stabilize the ferrous $\left(\mathrm{Fe}^{2+}\right)$ state, we prepared iron

496 as a $1: 10$ ratio of sodium ascorbate: $\mathrm{FeCl}_{2}$ immediately prior to the experiment. To determine the

497 full extent of the calcein quenching response, $10 \mu \mathrm{M}$ of the divalent cation ionophore calcimycin

498 (Sigma) was added at the end of each experiment. Transport data was normalized to the mean

499 baseline fluorescence intensity prior to addition of ion.

\section{Hepcidin binding assays}

502 Fluorescence polarization measurements were performed using rhodamine-green labeled

503 hepcidin (RhoG-hepcidin) ${ }^{25}$. For FPN saturation binding experiments, samples were prepared in

504 a black 384-well plate (Greiner) containing 0-1 $\mu \mathrm{M}$ of nanodisc reconstituted NW11-FPN and 5

505 nM RhoG-hepcidin in sample buffer comprising 20 mM HEPES pH 7.50, $100 \mathrm{mM} \mathrm{NaCl}$, and

506 supplemented with $\mathrm{FeCl}_{2}, \mathrm{CoCl}_{2}$ or $\mathrm{MnCl}_{2}$ as indicated. For ion stimulation experiments, $100 \mathrm{nM}$

507 NW11-FPN and $5 \mathrm{nM}$ RhoG-Hepcidin was mixed with $0-600 \mu \mathrm{M}$ of $\mathrm{CoCl}_{2}$. For Fab binding

508 experiments, $100 \mathrm{nM}$ NW11-FPN and $5 \mathrm{nM}$ RhoG-Hepcidin was mixed with $0-3 \mu \mathrm{M}$ of

509 Fab45D8 in sample buffer containing $10 \mu \mathrm{M} \mathrm{CoCl}_{2}$. Binding reactions were equilibrated for 60 
510 min at RT, and fluorescence polarization was recorded on a Biotek Synergy H4 (Agilent) in

511 polarization mode using fixed bandpass filters with $\lambda_{\mathrm{ex}}$ of $484 \mathrm{~nm}$ and $\lambda_{\mathrm{em}}$ of $520 \mathrm{~nm}$.

513 Analytical fluorescence size exclusion chromatography (FSEC) was performed by mixing $25 \mu \mathrm{g}$

514 of NW11-FPN with $2 x$ fold molar excess of RhoG-Hepcidin in sample buffer comprised of 20

$515 \mathrm{mM}$ HEPES (pH 7.50), $100 \mathrm{mM} \mathrm{NaCl}$ and $10 \mu \mathrm{M} \mathrm{CoCl}_{2}$. Samples were incubated for $20 \mathrm{~min}$ on

516 ice and $1.5 \times$ molar excess of Fab45D8, or sample buffer, was added followed by incubation for

51730 min on ice. For homologous competition, $1 \mu \mathrm{M}$ NW11-FPN was mixed with $30 \mu \mathrm{M}$ unlabelled

518 hepcidin (Bachem) in sample buffer comprised of $20 \mathrm{mM} \mathrm{HEPES} \mathrm{(pH} \mathrm{7.50),} 100 \mathrm{mM} \mathrm{NaCl}$ and

$51910 \mu \mathrm{M} \mathrm{CoCl}$, and incubated for 30 min on ice. Then $2 \mu \mathrm{M}$ RhoG-Hepcidin was added and the

520 sample incubated for $30 \mathrm{~min}$ on ice. Samples were injected on a Superdex 200 Increase 10/300

521 GL column (GE Lifesciences) pre-equilibrated in $20 \mathrm{mM} \mathrm{HEPES} \mathrm{pH} \mathrm{7.50,} 100 \mathrm{mM} \mathrm{NaCl}$, and 10

$522 \mu \mathrm{M} \mathrm{CoCl}_{2}$. RhoG-Hepcidin fluorescence was recorded using an FP-1520 Intelligent

523 Fluorescence Detector (Jasco) with $\lambda_{\mathrm{ex}}$ of $493 \mathrm{~nm}$ and $\lambda_{\mathrm{em}}$ of $524 \mathrm{~nm}$.

524

525 Cryo-EM Sample Preparation and Data Collection

526 Nanodisc-reconstituted apo-FPN was mixed with 1.15 molar excess of Fab45D8 and incubated on ice for $30 \mathrm{~min}$. The complex was purified by size-exclusion chromatography over a Superdex S200 Increase 10/300 GL column (GE Healthcare) equilibrated with 20 mM HEPES pH 7.50, $100 \mathrm{mM} \mathrm{NaCl}$. For $\mathrm{Co}^{2+} /$ hepcidin samples, $600 \mu \mathrm{M} \mathrm{CoCl}_{2}$ and $30 \mu \mathrm{M}$ hepcidin (Bachem) was

530 added to nanodisc-reconstituted FPN and incubated for 20 minutes on ice prior to addition of 531 Fab45D8. The resulting complex was purified over size-exclusion chromatography as for the 532 apo sample but with the addition of $100 \mu \mathrm{M} \mathrm{CoCl}_{2}$ in the chromatography buffer. Collected 533 fractions were supplemented with fresh hepcidin to $30 \mu \mathrm{M}$. For both preparations, fractions 534 containing the nanodisc-FPN-Fab45D8 complex were concentrated to $\sim 3 \mathrm{mg} / \mathrm{ml}$ on a Vivaspin 535 50-kDa MWCO concentrator and freshly used for electron microscopy.

537 For high-resolution cryo-EM, the apo-FPN-Fab45D8 complex was diluted to $0.0375 \mathrm{mg} / \mathrm{mL}$ in 20 $538 \mathrm{mM}$ HEPES pH 7.5, $100 \mathrm{mM} \mathrm{NaCl}$ directly prior to vitrification, and $2 \mu \mathrm{L}$ sample was applied to 539 glow-discharged gold holey carbon 1.2/1.3 300-mesh grids (Quantifoil) coated in-house with 540 graphene oxide ${ }^{5-57}$. Grids were blotted for 2-4 seconds at 0 force and 10 seconds wait time 541 before being plunge vitrified in liquid ethane using a MarkIV Vitrobot (ThermoFisher). The 542 blotting chamber was maintained at $22^{\circ} \mathrm{C}$ and $100 \%$ humidity during freezing. 


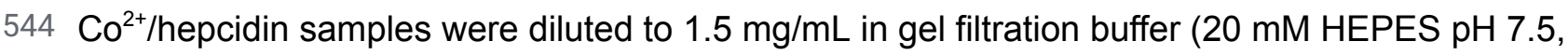

$545100 \mathrm{mM} \mathrm{NaCl}, 100 \mu \mathrm{M} \mathrm{CoCl}_{2}$ ) before vitrification. Grids were blotted for 3 seconds at 0 force

546 and 5 seconds wait time before being plunge vitrified in liquid ethane using a MarkIV Vitrobot

547 (ThermoFisher). The blotting chamber was maintained at $22^{\circ} \mathrm{C}$ and $100 \%$ humidity during

548 freezing.

550 FPN-Fab45D8 and Co2+/hepcidin-FPN-Fab45D8 movies were collected using a Titan Krios

551 (ThermoFisher) outfitted with a K3 camera and Bioquantum energy filter (Gatan). The K3

552 detector was operated in superresolution mode and the energy filter slit width was set to $20 \mathrm{eV}$.

553 Movies were collected at a nominal magnification of $105,000 x$, physical pixel size $0.834 \AA /$ pix,

554 with a $70 \mu \mathrm{m} \mathrm{C2}$ aperture and $100 \mu \mathrm{m}$ objective aperture at a dose rate of $8 \mathrm{e} / \mathrm{pixel} / \mathrm{second}$. A

555 total dose of $66 \mathrm{e}^{-/} / \AA^{2}$ was collected as a 120 -frame movie, resulting in a 6-second movie with

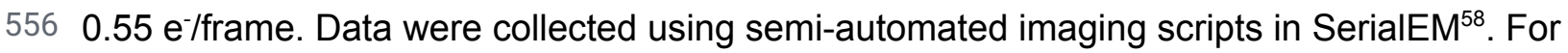

557 FPN-Fab45D8, 5009 movies were collected using a 3×3 image shift pattern at $0^{\circ}$ tilt and 406

558 movies were collected on-axis with a $30^{\circ}$ stage tilt in two separate data collection sessions. For

559 Co2+/hepcidin-FPN-Fab45D8, 4395 movies were collected at $0^{\circ}$ tilt in a single data collection

560 session.

561

\section{Cryo-EM Image Processing}

563 For FPN-Fab45D8, data were motion corrected and 2x binned on-the-fly using MotionCor2 ${ }^{59}$ in

564 the SCIPION pipeline ${ }^{60}$. Motion corrected micrographs were imported into cryoSPARC ${ }^{61}$ and

565 RELION $^{62}$ and contrast transfer function parameters were calculated using CTFFIND4 ${ }^{63}$. CTF

566 information for tilted images were estimated using patch CTF estimation in cryoSPARC.

567 138,314 particles were selected from 497 micrographs using the Blob picker in cryoSPARC. 2D

568 class averages were generated after extracting the putative particles with a 300-pixel box and

569 binning to 64 pixels. Six of these averages were used as templates for further particle picking.

570 Template picking yielded 4,737,795 particles. These were split into 6 groups to increase speed

571 of processing, extracted in a 300-pixel box, and binned to 64 pixels. 2D classification was run in

572 cryoSPARC with default settings except: number of 2D classes 200, initial classification

573 uncertainty factor 4 , number of online-EM iterations 40 , batch size per class 300 . Objectively

574 "good" (showing clear Fab and receptor density) class averages were selected and exported to

575 RELION format using csparc.py ${ }^{64}$. Class averages that were not classified as "good", but were 
576 not clearly ice contamination or graphene oxide edges, were run through a second round of 2D

577 classification with default settings except: number of 2D classes 200, number of online-EM

578 iterations 40, batch size per class 300. All "saved" class averages from the second rounds of 2D

579 classification in cryoSPARC selected and exported to RELION format using csparc.py. Particles

580 were extracted from CTF-corrected images in RELION at a box size of 300 pixels, binned to 128

581 pixels. 1,326,130 particles, in three groups (detailed in Supplementary Fig. 3) were classified in

582 3D with image alignment in RELION using an initial model generated in cryoSPARC from

58380,000 particles collected on a Talos Arctica filtered to $40 \AA$, C1 symmetry, a regularization

584 parameter of 4 , for 30-35 iterations with no mask. Particles from classes with resolved

585 transmembrane (TM) helices were selected, extracted in a 300-pixel box, and imported back

586 into cryoSPARC. Non-uniform refinement was run with default settings and no resolution limit,

587 resulting in angle and shift assignments for 850,000 particles. These particles were

588 subsequently exported to RELION format using csparc.py and run through 3D classification

589 without image alignment in RELION. Four of the 12 classes were selected, imported into

590 RELION, run through non-uniform alignment with an automatically generated mask, and refined

591 to a reported global resolution of $3.2 \AA$. The resulting map showed clear signs of mild preferred

592 orientation (Supplementary Fig. 3). The particles were exported into RELION format using

593 csparc.py, converted into an image stack, and imported into cisTEM ${ }^{65}$ as a refinement package.

594 The particles were reconstructed and half-maps were generated using the "generate 3D"

595 command. These half-maps, as well as the half-maps from cryoSPARC were run through our

596 lab's directional Fourier shell correlation (dFSC) program ${ }^{66}$ clearly showing a more distributed

597 range of views in the map generated by cisTEM. Maps were sharpened in RELION. Resolutions

598 are reported using the FSC $=0.143$ cut-off $^{67}$ and were estimated in cryoSPARC and cisTEM.

600 For $\mathrm{Co}^{2+} /$ hepcidin-FPN-Fab45D8, data were motion corrected and 2x binned on-the-fly using

601 MotionCor $2^{59}$ in the SCIPION pipeline ${ }^{60}$. Motion corrected micrographs were imported into

602 cryoSPARC $^{61}$ and contrast transfer function parameters were calculated using CTFFIND4 ${ }^{63}$.

$6033,753,516$ particles were selected from 4395 micrographs using the template picker in

604 cryoSPARC with 2D averages from the apo-FPN dataset as templates. These particles were

605 extracted in a 360-pixel box, and binned to 64 pixels. 2D classification was run in cryoSPARC

606 with default settings except: number of 2D classes 200 , initial classification uncertainty factor 4 ,

607 number of online-EM iterations 40, batch size per class 300. Objectively "good" (showing clear

608 Fab and receptor density) class averages were selected for 3D classification. Class averages 
609 that were not classified as "good", but were clearly not ice contamination, were run through a

610 second round of 2D classification with default settings except: number of 2D classes 200,

611 number of online-EM iterations 40, batch size per class 300. All "saved" class averages from the

612 second rounds of 2D classification in cryoSPARC were sent to 3D classification. These particles

613 were subjected to two rounds of heterogeneous refinement in cryoSPARC that serves as a

614 "trash collector". Four initial models were used, three generated from an early round of ab initio

615 model generation and our final apo-FPN-Fab45D8 structure. All initial models were filtered to

$61630 \AA$ before refinement. Particles were unbinned and a final heterogeneous refinement was

617 performed with three good initial models of apo-FPN-Fab45D8. Non-uniform refinement was run

618 with default settings and no resolution limit, on the most populated class resulting in angle and

619 shift assignments for 310,647 particles. The particles were exported into RELION format using

620 csparc.py, converted into an image stack, and imported into cisTEM ${ }^{65}$ as a refinement package.

621 The particles were reconstructed and half-maps were generated using the "generate 3D"

622 command. These half-maps, as well as the half-maps from cryoSPARC were run through our

623 lab's directional Fourier shell correlation (dFSC) program ${ }^{66}$ clearly showing a more distributed

624 range of views in the map generated by cisTEM. Maps were sharpened in cisTEM. Resolutions

625 are reported using the FSC $=0.143$ cut-off $^{67}$ and were estimated in cryoSPARC and cisTEM.

\section{Model building and refinement}

628 For apo-FPN, a homology model of human FPN in the outward open state was built using

629 Modeller $^{68}$, with a previously determined X-ray crystal structure of outward open bbFPN (PDB

630 ID: $5 A Y N)^{23}$ as a template. After truncating putatively flexible regions (N-terminus, ICL3, ECL5,

631 and C-terminus), the resulting model was fit into the $3.2 \AA$ cryo-EM map of FPN:Fab45D8 using

632 Chimera $^{69}$. The initial template was manually rebuilt in $\operatorname{Coot}^{52}$ and iteratively refined with real

633 space refinement implemented in Phenix ${ }^{53}$. Model geometry was assessed using MolProbity ${ }^{54}$.

634 Further validation was performed with EMRinger ${ }^{70}$ to compare the map and final model.

635 Map-to-model FSCs were calculated within Phenix. Figures were prepared in Chimera ${ }^{69}$ and 636 PyMol.

638 To dock hepcidin into the $\mathrm{Co}^{2+} /$ hepcidin-bound and apo-FPN difference density, we used a 639 previously determined X-ray crystal structure of hepcidin bound to a neutralizing Fab as a 640 starting mode ${ }^{21}$. Hepcidin, without the first two residues, was manually placed within the 641 difference density in Coot, then real-space refined to conform to the difference density 
642 with maintaining the disulfide connectivity and secondary structure observed in the

643 starting model. The resulting model docked to FPN has an overall RMSD of $1.2 \AA$

644 compared to the starting model for regions with defined secondary structure.

646 Molecular dynamics simulations

647 The structure of the outward-open apo conformation of human FPN was used as the starting 648 coordinates for all simulations. Three different conditions were simulated (Supplementary Table 649 3): (1) the iron-absent condition, where no iron was added; (2) the iron-bound condition, where 650 an $\mathrm{Fe}^{2+}$ ion was placed in the proposed iron binding site $5.6 \AA$ from D325 $\alpha$-carbon, $6.9 \AA$ from 651 D504 a-carbon, and 7.8 A from H507 a-carbon; (3) the iron-in-bulk-solvent condition, where 15 $652 \mathrm{Fe}^{2+}$ ions were placed randomly in the water box outside the protein using Dabble ${ }^{71}$.

654 Simulation coordinates were prepared by removing non-FPN molecules from the initial 655 structure. Prime (Schrödinger) was used to model missing side chains, and neutral acetyl and 656 methylamide groups were added to cap protein termini. The unresolved loops between 657 TM6-TM7 and TM9-TM10 (residues 239-290 and 394-451 respectively, inclusively) were not 658 modeled. The termini surrounding these loops were capped. PropKa was used to determine the 659 dominant protonation state of all titratable residues at $\mathrm{pH} 7^{72,73}$. The structure was internally 660 hydrated using Dowser ${ }^{74}$. Dabble was used to additionally fill the extracellular cavity ${ }^{71}$. The 661 structure was aligned using the Orientation of Proteins in Membranes (OPM) server ${ }^{75}$.

663 Using Dabble, the protein was inserted into a pre-equilibrated

664 1-palmitoyl-2-oleoylphosphatidylcholine (POPC) membrane bilayer. For all simulations except 665 condition three (iron in bulk solvent), sodium and chloride ions were added at $150 \mathrm{mM}$ to 666 neutralize the system. For condition three, chloride ions were added to neutralize the system 667 resulting in a concentration of $108 \mathrm{mM}$. A periodic box was used with dimensions $90 \times 90 \AA$ in 668 the $x-y$ plane and a water buffer of $10 \AA$ above and below the protein to the periodic boundary. 669 We used the CHARMM36m parameters for lipids, proteins, sodium and chloride ions, and the 670 TIP3P model for waters ${ }^{76-78}$. The $\mathrm{Fe}^{2+}$ Lennard-Jones parameters were obtained from Li et. al.'s 671 compromise model ${ }^{79}$.

672

673 All simulations were run on a single Graphical Processing Unit (GPU) using the Amber18

674 Compute Unified Device Architecture (CUDA) version of particle-mesh Ewald molecular 
675 dynamics (PMEMD) ${ }^{80,81}$. For each condition, 6 replicates were run. For each independent 676 replicate, the system was minimized with 500 steps of steepest descent followed by 500 steps 677 of conjugate gradient descent three times. 10 and $5 \mathrm{kcal} \mathrm{mol}^{-1}-\AA^{2}$ harmonic restraints were used 678 on the protein, lipid, and $\mathrm{Fe}^{2+}$ ions for the first and second minimization, respectively. $1 \mathrm{kcal}$ $679 \mathrm{~mol}^{-1}-\AA^{2}$ harmonic restraints were used on the protein and $\mathrm{Fe}^{2+}$ ions for the final minimization.

680 The system was then heated from $0 \mathrm{~K}$ to $100 \mathrm{~K}$ over $12.5 \mathrm{ps}$ in the NVP ensemble with a 681 Langevin thermostat and harmonically restraining the protein heavy atoms and $\mathrm{Fe}^{2+}$ ions with a 682 restraint of $10 \mathrm{kcal} \mathrm{mol}^{-1} \dot{\mathrm{A}}^{2}$. The system was further heated with the same restraints from $100 \mathrm{~K}$ 683 to $310 \mathrm{~K}$ in the NPT ensemble over $125 \mathrm{ps}$. The system was equilibrated with harmonic 684 restraints on protein heavy atoms and $\mathrm{Fe}^{2+}$ ions for $30 \mathrm{~ns}$. The restraint strength started at $5 \mathrm{kcal}$ $685 \mathrm{~mol}^{-1} \dot{\mathrm{A}}^{2}$ and was reduced by $1 \mathrm{kcal} \mathrm{mol}^{-1} \AA^{2}$ every $2 \mathrm{~ns}$ for the first $10 \mathrm{~ns}$ and then by $0.1 \mathrm{kcal}$ $686 \mathrm{~mol}^{-1}-\dot{\mathrm{A}}^{2}$ every $2 \mathrm{~ns}$ for the final $20 \mathrm{~ns}$. Production simulations were performed at $310 \mathrm{~K}$ and 1 687 bar using the NPT ensemble, a Langevin thermostat and a Monte Carlo barostat. Every 200 ps 688 snapshots were saved. All simulations were run for at least $2.2 \mu \mathrm{s}$. These simulations used a 689 4-fs time step with hydrogen mass repartitioning ${ }^{82}$. Bond lengths to hydrogen atoms were 690 constrained using SHAKE ${ }^{82,83}$. Non-bonded interactions were cut off at $9 \AA$.

\section{Simulation Analysis Methods}

693 MD snapshots were reimaged every $1 \mathrm{~ns}$ and centered using CPPTRAJ package in 694 AmberTools $18^{84}$. Simulations were visualized using Visual Molecular Dynamics and figures 695 prepared in $\mathrm{PyMOL}^{85}$. Time traces from simulation were smoothed using a moving average with 696 a window size of 15 ns unless otherwise indicated and visualized with the PyPlot package from 697 Matplotlib. For all analysis in the manuscript that required structural alignment, we aligned to the 698 initial Ferroportin structure using the backbone atoms of residues 26-116, 127-228, 308-483, 699 and 492-543.

701 To investigate the localization of $\mathrm{Fe}^{2+}$ ions, the iron-in-bulk-solvent simulations (condition 3 ) were 702 analyzed. To visualize the density of $\mathrm{Fe}^{2+}$ ions, the position of $\mathrm{Fe}^{2+}$ ions was recorded every 10 703 ns for each of the 6 simulation replicates, each $2 \mu \mathrm{s}$ in length. Each $\mathrm{Fe}^{2+}$ ion position was then 704 drawn as a point superimposed on the starting structure (Fig. 3d). To quantify the binding 705 events, the distance between iron and the closest side chain oxygen atom on D325 was 706 measured. This distance was graphed over $1.2 \mu \mathrm{s}$, including the equilibration time (Fig. 3d). 
708 To investigate the dynamics and conformation of the TM7b region, the iron-absent (condition 1)

709 and iron-bound (condition 2) simulations were compared. The TM1-TM7b distance was

710 measured using distance between the Ca of V51 and the Ca of Y333. For each simulation, we

711 calculated the average of the distance over $2.2 \mu \mathrm{s}$, excluding equilibration. The average over

712 the simulations for each condition was plotted with error bars representing the standard error of

713 the mean (s.e.m.) (Supplementary Fig. 7). The dynamics of D325 were also investigated. The

714 dynamics were visualized by overlaying representative frames showing the movement of D325,

715 the binding site, and TM7b. For iron-absent simulations, frames from a single replicate at 200,

716 350, 500, and 550 ns were overlaid (Supplementary Fig. 7). For iron-bound simulations, frames

717 from a single replicate at 200, 500, 725, 1000 ns were overlaid (Supplementary Fig. 7). The

718 conformational range of D325 was quantified by measuring the distance between Cy of D325

719 and C $\beta$ of S47. This was visualized for one replicate for each condition over a time of $1 \mu \mathrm{S}$

720 inclusive of equilibration (Supplementary Fig. 7). For each independent replicate, the mean of

721 the Cy D325 - C $\beta$ S47 distance was calculated over $2.2 \mu \mathrm{s}$. For each condition, the average

722 over the replicates was plotted with error bars representing the s.e.m. (Supplementary. Fig. 7).

723 The flexibility of D325 was quantified by calculating the root-mean-square fluctuation (RMSF) of

724 the side-chain atoms of D325 using an in-house script (Supplementary Fig. 7). Statistical

725 significance was determined using the Mann-Whitney $U$ test.

728 Data Availability

729 All data generated or analyzed during this study are included in this published article and its

730 Supplementary Information. Crystallographic coordinates and structure factors for the Fab45D8

731 complex have been deposited in the Protein Data Bank under accession code 6W4V.

732 Coordinates for Fab45D8-FPN complex have been deposited in the Protein Data Bank under

733 accession code 6W4S and the maps have been deposited in the Electron Microscopy Data

734 Bank under accession code 21539. Coordinates for the FPN-Co ${ }^{2+}$-hepcidin-Fab45D8 complex

735 have been deposited in the Protein Data Bank under accession code 6WBV and the maps have

736 been deposited in the Electron Microscopy Data Bank under accession code 21599.

738 Conflict of Interest

739 Tara Arvedson is employed by Amgen and reports Amgen stock. None of the other authors

740 report conflicts of interest. 


\section{REFERENCES}

742 1. Drakesmith, H., Nemeth, E. \& Ganz, T. Ironing out Ferroportin. Cell Metab. 22, 777-787 (2015).

743 2. Donovan, A. et al. The iron exporter ferroportin/Slc40a1 is essential for iron homeostasis. Cell Metab.

$744 \quad 1,191-200(2005)$.

745 3. Donovan, A. et al. Positional cloning of zebrafish ferroportin1 identifies a conserved vertebrate iron

746 exporter. Nature 403, 776-781 (2000).

747 4. Knutson, M. D., Oukka, M., Koss, L. M., Aydemir, F. \& Wessling-Resnick, M. Iron release from

748 macrophages after erythrophagocytosis is up-regulated by ferroportin 1 overexpression and

749 down-regulated by hepcidin. Proc. Natl. Acad. Sci. U. S. A. 102, 1324-1328 (2005).

750 5. Ward, D. M. \& Kaplan, J. Ferroportin-mediated iron transport: expression and regulation. Biochim.

$751 \quad$ Biophys. Acta 1823, 1426-1433 (2012).

752 6. Aschemeyer, S. et al. Structure-function analysis of ferroportin defines the binding site and an

753 alternative mechanism of action of hepcidin. Blood, The Journal of the American Society of

$754 \quad$ Hematology 131, 899-910 (2018).

755 7. Nemeth, E. et al. Hepcidin regulates cellular iron efflux by binding to ferroportin and inducing its

756 internalization. Science 306, 2090-2093 (2004).

757 8. De Domenico, I. et al. The molecular mechanism of hepcidin-mediated ferroportin down-regulation.

758 Mol. Biol. Cell 18, 2569-2578 (2007).

759 9. Qiao, B. et al. Hepcidin-induced endocytosis of ferroportin is dependent on ferroportin ubiquitination.

760 Cell Metab. 15, 918-924 (2012).

761 10. Ross, S. L. et al. Molecular mechanism of hepcidin-mediated ferroportin internalization requires

762 ferroportin lysines, not tyrosines or JAK-STAT. Cell Metab. 15, 905-917 (2012).

763 11. Roetto, A. et al. Mutant antimicrobial peptide hepcidin is associated with severe juvenile

764 hemochromatosis. Nat. Genet. 33, 21-22 (2003).

765 12. De Domenico, I. et al. The molecular basis of ferroportin-linked hemochromatosis. Proc. Natl. Acad.

766 Sci. U. S. A. 102, 8955-8960 (2005). 
767 13. Drakesmith, $\mathrm{H}$. et al. Resistance to hepcidin is conferred by hemochromatosis-associated mutations

768 of ferroportin. Blood 106, 1092-1097 (2005).

769 14. Roy, C. N. et al. Hepcidin antimicrobial peptide transgenic mice exhibit features of the anemia of 770 inflammation. Blood 109, 4038-4044 (2007).

771 15. Ganz, T. \& Nemeth, E. The hepcidin-ferroportin system as a therapeutic target in anemias and iron 772 overload disorders. Hematology Am. Soc. Hematol. Educ. Program 2011, 538-542 (2011).

773 16. Manolova, V. et al. Oral ferroportin inhibitor ameliorates ineffective erythropoiesis in a model of $774 \quad \beta$-thalassemia. J. Clin. Invest. (2019) doi:10.1172/JCI129382.

775 17. Witcher, D. R. et al. LY2928057, an antibody targeting ferroportin, is a potent inhibitor of hepcidin 776 activity and increases iron mobilization in normal cynomolgus monkeys. (2013).

777

18. Crielaard, B. J., Lammers, T. \& Rivella, S. Targeting iron metabolism in drug discovery and delivery. Nat. Rev. Drug Discov. 16, 400-423 (2017).

19. Vlasveld, L. T. et al. Twenty Years of Ferroportin Disease: A Review or An Update of Published Clinical, Biochemical, Molecular, and Functional Features. Pharmaceuticals 12, (2019).

20. Nemeth, E. et al. The N-terminus of hepcidin is essential for its interaction with ferroportin: structure-function study. Blood 107, 328-333 (2006).

21. Jordan, J. B. et al. Hepcidin revisited, disulfide connectivity, dynamics, and structure. J. Biol. Chem. 284, 24155-24167 (2009).

22. Bonaccorsi di Patti, M. C. et al. A structural model of human ferroportin and of its iron binding site. FEBS J. 281, 2851-2860 (2014).

23. Taniguchi, R. et al. Outward- and inward-facing structures of a putative bacterial transition-metal transporter with homology to ferroportin. Nat. Commun. 6, 8545 (2015).

24. Deshpande, C. N. et al. Calcium is an essential cofactor for metal efflux by the ferroportin transporter family. Nat. Commun. 9, 3075 (2018).

25. Ross, S. L. et al. Identification of Antibody and Small Molecule Antagonists of Ferroportin-Hepcidin Interaction. Front. Pharmacol. 8, 838 (2017).

26. Wu, S. et al. Fabs enable single particle cryoEM studies of small proteins. Structure 20,582-592 
(2012).

27. Mitchell, C. J., Shawki, A., Ganz, T., Nemeth, E. \& Mackenzie, B. Functional properties of human ferroportin, a cellular iron exporter reactive also with cobalt and zinc. Am. J. Physiol. Cell Physiol. 306, C450-9 (2014).

28. Guellec, J. et al. Molecular model of the ferroportin intracellular gate and implications for the human iron transport cycle and hemochromatosis type 4A. FASEB J. 33, 14625-14635 (2019).

29. Sham, R. L. et al. Autosomal dominant hereditary hemochromatosis associated with a novel ferroportin mutation and unique clinical features. Blood Cells Mol. Dis. 34, 157-161 (2005).

30. Schimanski, L. M. et al. In vitro functional analysis of human ferroportin (FPN) and hemochromatosis-associated FPN mutations. Blood 105, 4096-4102 (2005).

31. Viprakasit, V. et al. Molecular Diagnosis of the First Ferroportin Mutation (C326Y) in the Far East Causing a Dominant Form of Inherited Iron Overload. (2004).

32. Mayr, R. et al. Identification of mutations in SLC40A1 that affect ferroportin function and phenotype of human ferroportin iron overload. Gastroenterology 140, 2056-63, 2063.e1 (2011).

33. Tortosa, V., di Patti, M. C. B., Brandi, V., Musci, G. \& Polticelli, F. An improved structural model of the human iron exporter ferroportin. Insight into the role of pathogenic mutations in hereditary hemochromatosis type 4. Bio-Algorithms and Med-Systems 13, 215-222 (2017). overload due to a novel mutation of ferroportin1 associated with parenchymal iron loading and cirrhosis. Journal of Hepatology vol. 40 710-713 (2004). Nat. Genet. 28, 213-214 (2001).

36. Arden, K. E. et al. A novel mutation in ferroportin1 is associated with haemochromatosis in a 817 Solomon Islands patient. Gut 52, 1215-1217 (2003).

818 37. Zhang, W. et al. A novel SLC40A1 p.Y333H mutation with gain of function of ferroportin: A recurrent cause of haemochromatosis in China. Liver Int. 39, 1120-1127 (2019).

820 38. Létocart, E. et al. A novel missense mutation in SLC40A1 results in resistance to hepcidin and 
confirms the existence of two ferroportin-associated iron overload diseases. Br. J. Haematol. 147, 379-385 (2009).

39. Callebaut, I. et al. Comprehensive functional annotation of 18 missense mutations found in suspected hemochromatosis type 4 patients. Hum. Mol. Genet. 23, 4479-4490 (2014).

40. Preza, G. C. et al. Minihepcidins are rationally designed small peptides that mimic hepcidin activity in mice and may be useful for the treatment of iron overload. J. Clin. Invest. 121, 4880-4888 (2011).

41. Rivard, S. R. et al. Autosomal dominant reticuloendothelial iron overload (HFE type 4) due to a new missense mutation in the FERROPORTIN 1 gene (SLC11A3) in a large French-Canadian family. Haematologica 88, 824-826 (2003).

42. Raszeja-Wyszomirska, J., Caleffi, A., Milkiewicz, P. \& Pietrangelo, A. Ferroportin-related haemochromatosis associated with novel Y64H mutation of the SCL40A1 gene. Prz Gastroentero/ 9, 307-309 (2014).

43. Clark, R. J. et al. Understanding the structure/activity relationships of the iron regulatory peptide hepcidin. Chem. Biol. 18, 336-343 (2011).

44. Smythe, M. L. et al. Hepcidin analogues and uses thereof. US Patent (2017).

45. Bourne, G. T., Smythe, M. L., Frederick, B. T. \& Vink, S. Hepcidin and mini-hepcidin analogues and uses therof. US Patent (2020).

46. Galesloot, T. E. et al. Serum hepcidin: reference ranges and biochemical correlates in the general population. Blood 117, e218-25 (2011).

47. Nasr, M. L. et al. Covalently circularized nanodiscs for studying membrane proteins and viral entry. Nat. Methods 14, 49-52 (2017).

842 48. Winter, G., Lobley, C. M. C. \& Prince, S. M. Decision making in xia2. Acta Crystallogr. D Biol. Crystallogr. 69, 1260-1273 (2013).

49. Kabsch, W. XDS. Acta Crystallogr. D Biol. Crystallogr. 66, 125-132 (2010).

50. McCoy, A. J. et al. Phaser crystallographic software. J. Appl. Crystallogr. 40, 658-674 (2007).

51. Aleman, F. et al. Immunogenetic and structural analysis of a class of HCV broadly neutralizing antibodies and their precursors. Proc. Natl. Acad. Sci. U. S. A. 115, 7569-7574 (2018). 
52. Emsley, P. \& Cowtan, K. Coot: model-building tools for molecular graphics. Acta Crystallogr. D Biol. Crystallogr. 60, 2126-2132 (2004).

53. Adams, P. D. et al. PHENIX: a comprehensive Python-based system for macromolecular structure solution. Acta Crystallogr. D Biol. Crystallogr. 66, 213-221 (2010).

54. Chen, V. B. et al. MolProbity: all-atom structure validation for macromolecular crystallography. Acta Crystallogr. D Biol. Crystallogr. 66, 12-21 (2010).

854 55. Cote, L. J., Kim, F. \& Huang, J. Langmuir- Blodgett assembly of graphite oxide single layers. J. Am. Chem. Soc. 131, 1043-1049 (2009).

56. Palovcak, E. et al. A simple and robust procedure for preparing graphene-oxide cryo-EM grids. J. Struct. Biol. 204, 80-84 (2018).

57. Wang, F. et al. Amino and PEG-amino graphene oxide grids enrich and protect samples for high-resolution single particle cryo-electron microscopy. J. Struct. Biol. 209, 107437 (2020).

58. Mastronarde, D. N. SerialEM: A Program for Automated Tilt Series Acquisition on Tecnai Microscopes Using Prediction of Specimen Position. Microsc. Microanal. 9, 1182-1183 (2003).

59. Zheng, S. Q. et al. MotionCor2: anisotropic correction of beam-induced motion for improved cryo-electron microscopy. Nat. Methods 14, 331-332 (2017).

60. de la Rosa-Trevín, J. M. et al. Scipion: A software framework toward integration, reproducibility and validation in 3D electron microscopy. J. Struct. Biol. 195, 93-99 (2016).

61. Punjani, A., Rubinstein, J. L., Fleet, D. J. \& Brubaker, M. A. cryoSPARC: algorithms for rapid unsupervised cryo-EM structure determination. Nat. Methods 14, 290-296 (2017).

62. Scheres, S. H. W. RELION: implementation of a Bayesian approach to cryo-EM structure determination. J. Struct. Biol. 180, 519-530 (2012).

63. Rohou, A. \& Grigorieff, N. CTFFIND4: Fast and accurate defocus estimation from electron

871 micrographs. J. Struct. Biol. 192, 216-221 (2015).

872 64. Asarnow, D., Palovcak, E. \& Cheng, Y. asarnow/pyem: UCSF pyem v0.5. (2019).

873 doi:10.5281/zenodo.3576630.

874 65. Grant, T., Rohou, A. \& Grigorieff, N. cisTEM, user-friendly software for single-particle image 
875 processing. Elife 7, (2018).

876 66. Dang, S. et al. Cryo-EM structures of the TMEM16A calcium-activated chloride channel. Nature $\mathbf{5 5 2}$, $877 \quad 426-429(2017)$.

878 67. Rosenthal, P. B. \& Henderson, R. Optimal determination of particle orientation, absolute hand, and 879 contrast loss in single-particle electron cryomicroscopy. J. Mol. Biol. 333, 721-745 (2003).

880 68. Eswar, N. et al. Comparative protein structure modeling using MODELLER. Curr. Protoc. Protein Sci. Chapter 2, Unit 2.9 (2007).

882 69. Goddard, T. D., Huang, C. C. \& Ferrin, T. E. Visualizing density maps with UCSF Chimera. J. Struct. Biol. 157, 281-287 (2007).

884 70. Barad, B. A. et al. EMRinger: side chain-directed model and map validation for 3D cryo-electron microscopy. Nat. Methods 12, 943-946 (2015).

71. Betz, R. Dabble. (2017). doi:10.5281/zenodo.836914.

72. Olsson, M. H. M., Søndergaard, C. R., Rostkowski, M. \& Jensen, J. H. PROPKA3: Consistent Treatment of Internal and Surface Residues in Empirical pKa Predictions. J. Chem. Theory Comput. 7, 525-537 (2011).

73. Søndergaard, C. R., Olsson, M. H. M., Rostkowski, M. \& Jensen, J. H. Improved Treatment of Ligands and Coupling Effects in Empirical Calculation and Rationalization of pKa Values. J. Chem. Theory Comput. 7, 2284-2295 (2011).

74. Morozenko, A. \& Stuchebrukhov, A. A. Dowser, a new method of hydrating protein structures. Proteins: Structure, Function, and Bioinformatics vol. 84 1347-1357 (2016).

895 75. Lomize, M. A., Lomize, A. L., Pogozheva, I. D. \& Mosberg, H. I. OPM: orientations of proteins in membranes database. Bioinformatics 22, 623-625 (2006).

76. Klauda, J. B. et al. Update of the CHARMM all-atom additive force field for lipids: validation on six lipid types. J. Phys. Chem. B 114, 7830-7843 (2010).

899 77. Huang, J. et al. CHARMM36m: an improved force field for folded and intrinsically disordered 900 proteins. Nat. Methods 14, 71-73 (2017).

901 78. Beglov, D. \& Roux, B. Finite representation of an infinite bulk system: Solvent boundary potential for 
902 computer simulations. J. Chem. Phys. 100, 9050-9063 (1994).

903 79. Li, P., Roberts, B. P., Chakravorty, D. K. \& Merz, K. M., Jr. Rational design of particle mesh Ewald

904 compatible Lennard-Jones parameters for+ 2 metal cations in explicit solvent. J. Chem. Theory

$905 \quad$ Comput. 9, 2733-2748 (2013).

906 80. Case, D. A. et al. AMBER 2018; 2018. University of California, San Francisco.

907 81. Salomon-Ferrer, R., Götz, A. W., Poole, D., Le Grand, S. \& Walker, R. C. Routine Microsecond

908 Molecular Dynamics Simulations with AMBER on GPUs. 2. Explicit Solvent Particle Mesh Ewald. J.

909 Chem. Theory Comput. 9, 3878-3888 (2013).

910 82. Hopkins, C. W., Le Grand, S., Walker, R. C. \& Roitberg, A. E. Long-Time-Step Molecular Dynamics

911 through Hydrogen Mass Repartitioning. J. Chem. Theory Comput. 11, 1864-1874 (2015).

912 83. Ryckaert, J.-P., Ciccotti, G. \& Berendsen, H. J. C. Numerical integration of the cartesian equations of 913 motion of a system with constraints: molecular dynamics of n-alkanes. J. Comput. Phys. 23, 327-341

914 (1977).

915 84. Roe, D. R. \& Cheatham, T. E., 3rd. PTRAJ and CPPTRAJ: Software for Processing and Analysis of 916 Molecular Dynamics Trajectory Data. J. Chem. Theory Comput. 9, 3084-3095 (2013).

917 85. Humphrey, W., Dalke, A. \& Schulten, K. VMD: visual molecular dynamics. J. Mol. Graph. 14, 33-8, $918 \quad$ 27-8 (1996). 